\title{
Evaluation of Delivery Analysis to Detect Intrafractional Motion during Tomotherapy
}

\section{Kazuo Tarutani1,2*, Masao Tanooka1,3, Keisuke Sano ${ }^{3}$, Okada Wataru ${ }^{1,3}$, Masayuki Fujiwara ${ }^{1}$, Koichiro Yamakado ${ }^{1}$}

${ }^{1}$ Department of Radiology, Hyogo College of Medicine, Nishinomiya, Japan

${ }^{2}$ Japan Organization of Occupational Health and Safety Kansai Rousai Hospital, Amagasaki, Japan

${ }^{3}$ Department of Radiotherapy, Takarazuka City Hospital, Takarazuka, Japan

Email: *tarutani.kazuo@gmail.com

How to cite this paper: Tarutani, K., Tanooka, M., Sano, K., Wataru, O., Fujiwara, M. and Yamakado, K. (2019) Evaluation of Delivery Analysis to Detect Intrafractional Motion during Tomotherapy. International Journal of Medical Physics, Clinical Engineering and Radiation Oncology, 8, 225-235.

https://doi.org/10.4236/ijmpcero.2019.84020

Received: October 28, 2019

Accepted: November 11, 2019

Published: November 14, 2019

Copyright $\odot 2019$ by author(s) and Scientific Research Publishing Inc. This work is licensed under the Creative Commons Attribution International License (CC BY 4.0).

http://creativecommons.org/licenses/by/4.0/

\begin{abstract}
The purpose of this study was to investigate the ability of a management system (Delivery Analysis: DA) to detect intrafractional motion during intensity-modulated radiation therapy (IMRT) in tomotherapy mode. Tomotherapy has made it possible to manage internal movements during treatment using software DA, which quantifies using the information of the passing dose obtained during the radiation treatment of patients. First, three treatment plans for the test were created (lumbar spine, prostate, and femur). Second, a pelvis phantom was moved in the $\mathrm{X}, \mathrm{Y}$, and $\mathrm{Z}$ directions, and a sinogram was acquired. The magnitudes of the movements were $3 \mathrm{~mm}, 5 \mathrm{~mm}$, and $10 \mathrm{~mm}$, respectively. Finally, the ability of DA to detect the motion was evaluated by comparing the sinogram obtained by moving the phantom with a reference sinogram obtained without movement. The sensitivity of DA could be detected with a shift amount of $3 \mathrm{~mm}$ (gamma analysis tolerance $0.3 \mathrm{~mm} / 0.3 \%$ ). The average gamma analysis of each direction at $0.3 \mathrm{~mm} / 0.3 \%$ tolerance at each treatment site was $96.1 \%$ for the prostate, $93.5 \%$ for the lumbar spine, and $94.4 \%$ for the femur. Additionally, the average gamma pass rate results for the pelvic phantom in the $\mathrm{X}, \mathrm{Y}, \mathrm{Z}$ directions for a $10 \mathrm{~mm}$ shift were $96.2 \%$, $96.3 \%$, and $95.9 \%$, respectively. DA is a powerful tool with high detection sensitivity and ability to detect body movement during treatment.
\end{abstract}

\section{Keywords}

Delivery Analysis (DA), Tomotherapy, Sinogram, Intra-Fraction Motion, Quality Assurance (QA)

\section{Introduction}

Intensity-modulated radiation therapy (IMRT) is a useful method that can in- 
crease dose to tumor tissue while decreasing dose to organs at risk [1] [2]. Tomotherapy (Accuray, Sunnyvale, CA, USA) is one of the modern radiotherapy systems which allow a continuous dose delivery in a helical fashion around the anatomical site to be treated [3].

Based on a small $6 \mathrm{MV}$ linear accelerator attached to a ring gantry that rotates around the patient as the patient moves around the bore, it is mechanically unique and ultimately produces a helical path for radiation dose delivery. In tomotherapy, this technologically advanced radiation dose delivery system provides pre-treatment quality assurance $(\mathrm{QA})$ which ensures the safety of treatment by performing delivery quality assurance (DQA) [4] [5]. However, this method has not been used during treatment to evaluate intrafractional motion.

In volume-modulated arc therapy (VMAT) using a linear accelerator, the various types of pre-treatment QA have led to an improvement in safety [6] [7] [8] [9] [10]. Nevertheless, although these validations improve VMAT's safety, errors during treatment still produce random and systematic uncertainty [11].

With traditional pre-treatment QA, it is impossible to predict mechanical errors, inappropriate movements or human errors that occur during treatment [12] [13]. Therefore, methods of dose assessment during treatment have been developed in multiple research facilities [14]-[18], and have been commercialized [19] [20]. Ford et al. [21] conducted a validation with an electronic portal imaging device (EPID) during treatment and found multiple errors with potentially major severity. This makes management during treatment very important.

In a tomotherapy delivery system, delivery analysis (DA) software is implemented during treatment. DA compares pretreatment and measured in-treatment data with information describing the planned treatment delivery.

Pretreatment assessment provides tools to quickly confirm that the multileaf collimator (MLC) has performed according to the treatment plan and assess any differences in MLC performance that may impact delivery.

In-treatment assessment monitors the consistency of delivery with particular sensitivity to patient settings and anatomical variations and patient motion during treatment. Tomotherapy can use this feature to enable management during treatment.

Management during treatment with an EPID with a linear accelerator generally uses integrated data with X-rays transmitted through the patient, which reduces the accuracy of intrafractional motion error detection [22]. However, when DA is used in tomotherapy, data are output as sinogram data, not integrated data. Therefore, in DA, it can be expected that error detectability during treatment can be further improved. To date, however, the clinical significance of differing levels of discrepancy in in-treatment assessment has not been evaluated.

Therefore, we investigated the motion detectability of the DA management system in-treatment during IMRT treatment with tomotherapy. 


\section{Materials and Methods}

\subsection{Tomotherapy Delivery System, Radixact}

Tomotherapy unit is designed to provide IMRT with $6 \mathrm{MV}$ photon beam and binary 64 MLC [3]. Radixact (Version.1.1.0.1: Accuray, Sunnyvale, CA, USA) is the latest generation of Tomotherapy delivery systems and represents a redesign of both gantry and treatment planning systems. In Radixact, new QA software is renewed and DA is implemented.

The unit provides both "Tomohelical" and "TomoDirect" modes. Tomotherapy units clinically used three different field widths of $1.0,2.5$, and $5.0 \mathrm{~cm}$, which were defined by jaws along the longitudinal direction.

\subsection{Delivery Analysis (DA)}

\subsubsection{Pre-Treatment Assessment}

Pre-treatment assessment is a tool to assess that collimation is being performed according to the treatment plan. This plan reconstructs a measured MLC leaf open time sinogram by analyzing the pulse-by-pulse data from the detector during delivery and measuring the individual leaf open times using the high-signal pulses for each delivered projection. This assessment also calculates the dose distribution based on the measured MLC leaf open time sinogram, the original plan, and the planning CT, and compares and analyzes the original planned dose and the recalculated dose based on the measured MLC leaf open time sinogram. Because this study was an evaluation during treatment, we excluded this pre-treatment study.

\subsubsection{In-Treatment Assessment}

In-treatment assessment is software that quantifies consistency interfraction motion during treatment using post patient detector signals obtained during patient radiation therapy (Figure 1). The purpose is to capture changes in the patient's anatomy or positional misalignments. The data collection method uses a detector to receive X-rays that have passed through the patient's body during treatment. As soon as the treatment is finished, the data are taken to a stand-alone workstation separate from the treatment system network. This is done automatically. In addition, the consistency of irradiation can be evaluated by managing daily data in a trend graph.

\subsection{Assessment Method}

We used Radixact and the software used DA Ver1.1. A commercial pelvic phantom (ExacTrac Verification Phantom, BrainLab, Feldkirchen, Germany) was used. The assessment method is shown in Figure 2. Treatment plan consisted of three parts (lumbar spine and prostate, femur). The pelvic phantom was moved in the $\mathrm{X}, \mathrm{Y}$, and $\mathrm{Z}$ directions, assuming a body movement for each plan. The magnitudes of the errors to be moved were $3 \mathrm{~mm}, 5 \mathrm{~mm}$, and $10 \mathrm{~mm}$, respectively. Radixact's table drive accuracy was $\pm 0.5 \mathrm{~mm}$ (display value unit: 0.1 $\mathrm{mm}$ ). The first irradiation was used as a reference. The phantom was before treatment moved on the assumption of body movement with respect to the base 


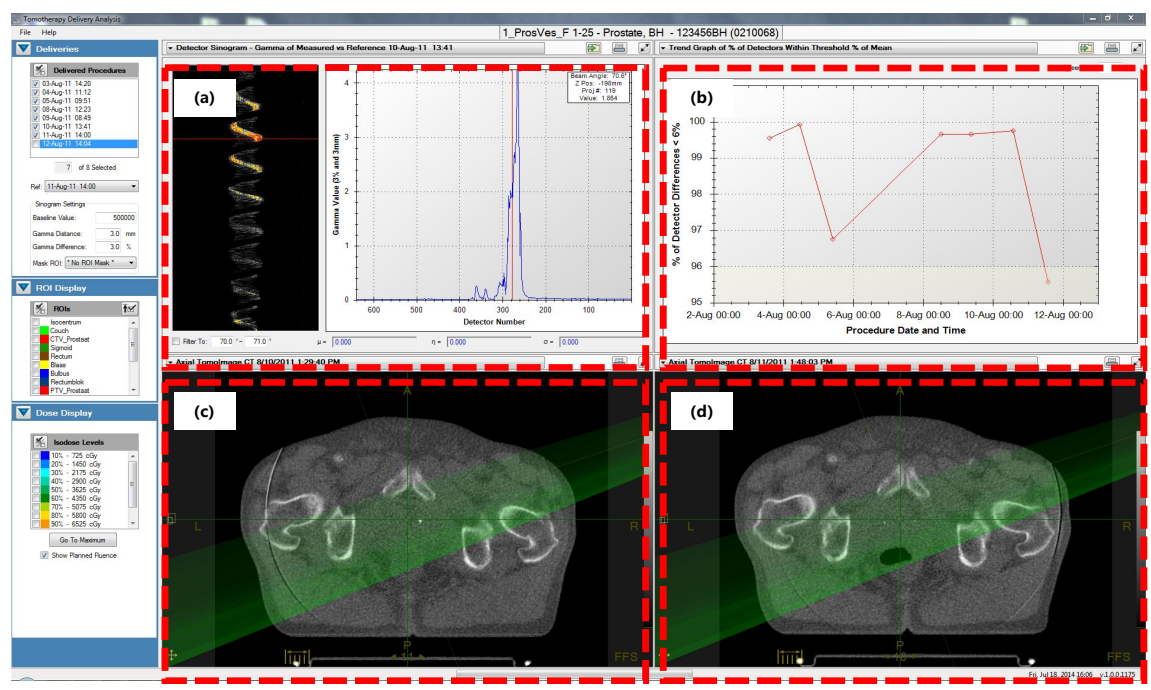

Figure 1. Example of analysis result by in-treatment assessment. (a) Detector sinogram views display the detector count distribution. A given point in the sinogram image corresponds to a particular detector position (horizontal axis) and a particular source position (vertical axis). The left of the view with the profile of the detector signal for the selected projection, which is indicated by the horizontal red line on the sinogram; (b) Trend View graphs plot changes in a selected value across a range of deliveries; (c) Reference MVCT image; (d) Evaluation MVCT image.

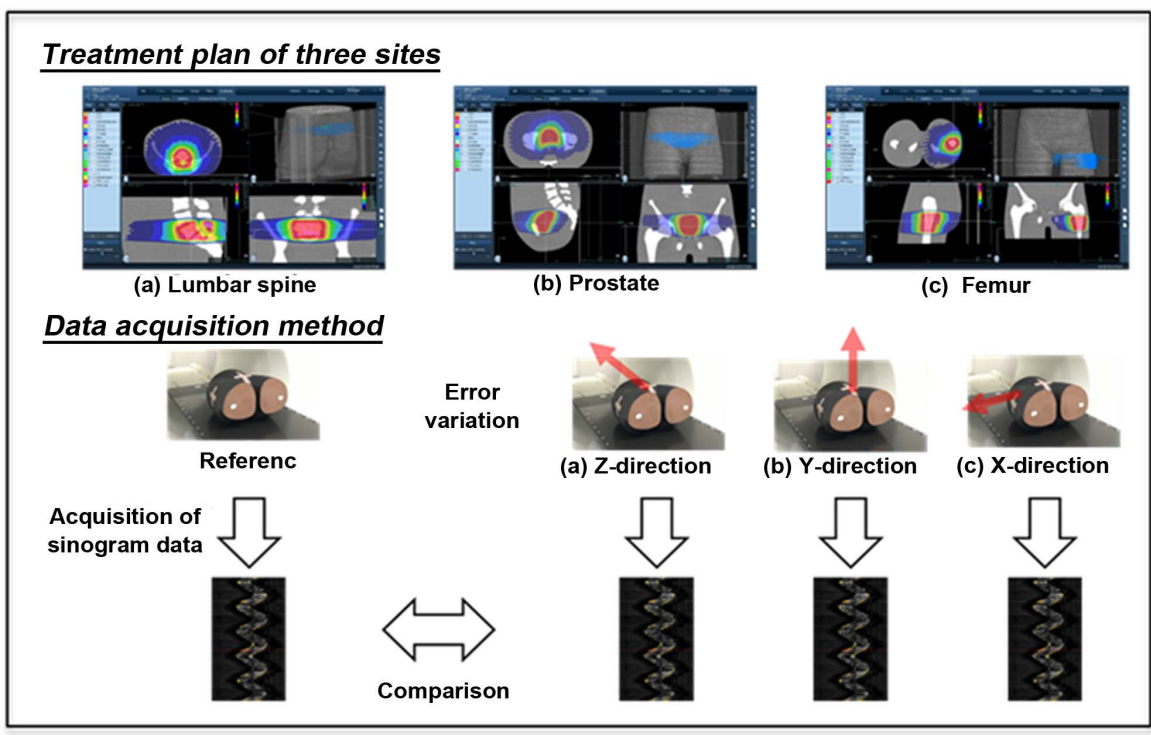

Figure 2. Error variation and data acquisition method. Treatment plan of three parts (lumbar spine, prostate, and femur). The pelvic phantom was moved in the $\mathrm{X}, \mathrm{Y}$, and $\mathrm{Z}$ directions, magnitudes of the errors to be moved were $3 \mathrm{~mm}$ and $5 \mathrm{~mm} 10 \mathrm{~mm}$, respectively, and sinogram data is acquired and evaluated by gamma analysis. Red arrow is indicates the shifted direction.

sinogram acquired first, and the acquired sinograms were compared. We used gamma analysis [23] as a comparison method. To confirm the sensitivity of gamma analysis tolerances, the setting values were changed to $3 \mathrm{~mm} / 3 \%, 2$ $\mathrm{mm} / 3 \%, 2 \mathrm{~mm} / 2 \%, 1 \mathrm{~mm} / 1 \%, 0.5 \mathrm{~mm} / 0.5 \%, 0.3 \mathrm{~mm} / 0.3 \%$. We also compared 
the analysis results.

In summary, $3 \mathrm{~mm}, 5 \mathrm{~mm}$, and $10 \mathrm{~mm}$ are moved in the $\mathrm{X}, \mathrm{Y}$, and $\mathrm{Z}$ directions for each part of the lumbar spine, prostate, and femur, and sinogram data was acquired and evaluated by gamma analysis. Also, the influence of the result of the gamma analysis which made variable the tolerance value for each relationship was investigated.

\section{Results}

Table 1 shown shows the results of gamma analysis in which the pelvic phantom was moved to $3 \mathrm{~mm}, 5 \mathrm{~mm}$, and $10 \mathrm{~mm}$ for each site. The measurement was performed five times and the average mean value was shown. The variation of the five measurements was small and the standard deviation was negligible. From this table, it is impossible to detect an error of movement at a tolerance of $3 \mathrm{~mm} / 3 \%$. However, when the tolerance is $0.3 \mathrm{~mm} / 0.3 \%$ and the shift is a $3 \mathrm{~mm}$ error, the result of gamma analysis at the lowest sensitivity is $94.0 \%$ for the prostate and $93.3 \%$ for the and $93.8 \%$ for the femur in and the lumbar spine, respectively. Therefore, in-treatment motion proved to be detectable.

\subsection{Influence of Result by Movement Amount}

The relationship between the shift amount of the pelvic phantom and the tolerance of gamma analysis is shown (Figure 3 ). The tolerance did not change significantly up to $2 \mathrm{~mm} / 2 \%$. When the tolerance was lowered to $1 \mathrm{~mm} / 1 \%$, the results were affected.

In addition, the amount of movement of the pelvic phantom was lower in gamma passing rate when it was moved by $10 \mathrm{~mm}$ compared to $3 \mathrm{~mm}$. Therefore, it was proved that the pass rate decreases in proportion to the magnitude of the shift amount of the pelvic phantom.

When the tolerance was $0.3 \mathrm{~mm} / 0.3 \%$, the average gamma pass rate was $94.7 \%, 92.2 \%$, and $89.9 \%$ when the shift amount of the pelvis phantom was 3 $\mathrm{mm}, 5 \mathrm{~mm}$, and $10 \mathrm{~mm}$, respectively. DA was able to detect a $3 \mathrm{~mm}$ shift.

\subsection{Influence of Result by Movement Direction}

The following shows the relationship between the movement direction and the result of gamma analysis (Figure 4). The average gamma pass rate results for the pelvic phantom for the $\mathrm{X}, \mathrm{Y}$, and $\mathrm{Z}$ directions for a $10 \mathrm{~mm}$ shift were $96.2 \%$, $96.4 \%$, and $95.9 \%$, respectively. No difference was found in the direction of movement.

\subsection{Influence of Result by Each Treatment Site}

Figure 5 shows the result of the pass rate of gamma analysis to tolerance at each treatment site. The average gamma analysis for each direction at $0.3 \mathrm{~mm} / 0.3 \%$ tolerance was $96.1 \%$ for the prostate, $93.5 \%$ for the lumbar spine, and $94.4 \%$ for the femur. It was confirmed that the sensitivity of the prostate was little lower than other parts. 
Table 1. The mean value of the gamma analysis (\%) that was performed five times and its standard deviation (\%). Results of gamma analysis with different tolerance values and phantom movement values for each part (prostate, lumbar vertebra, femur).

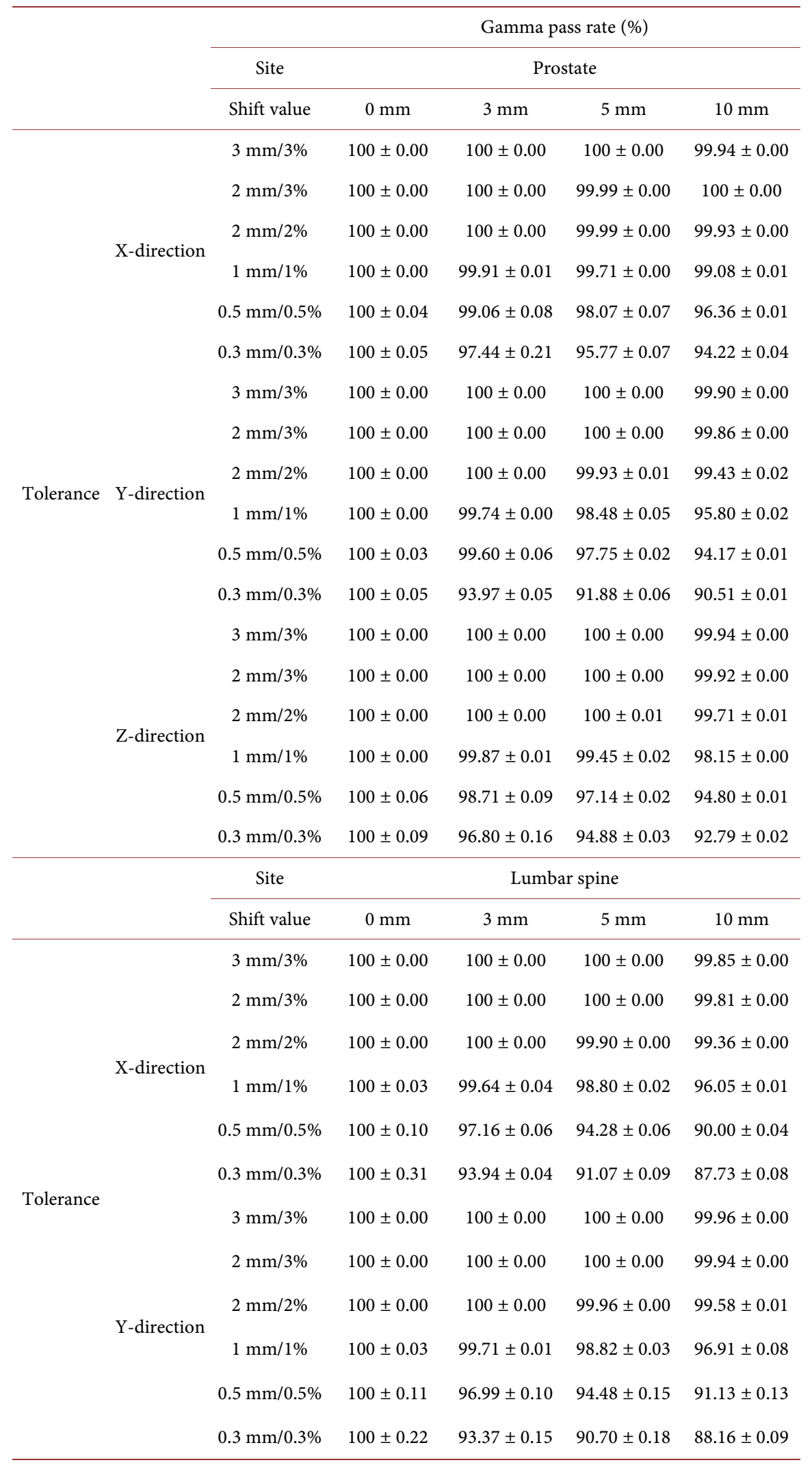




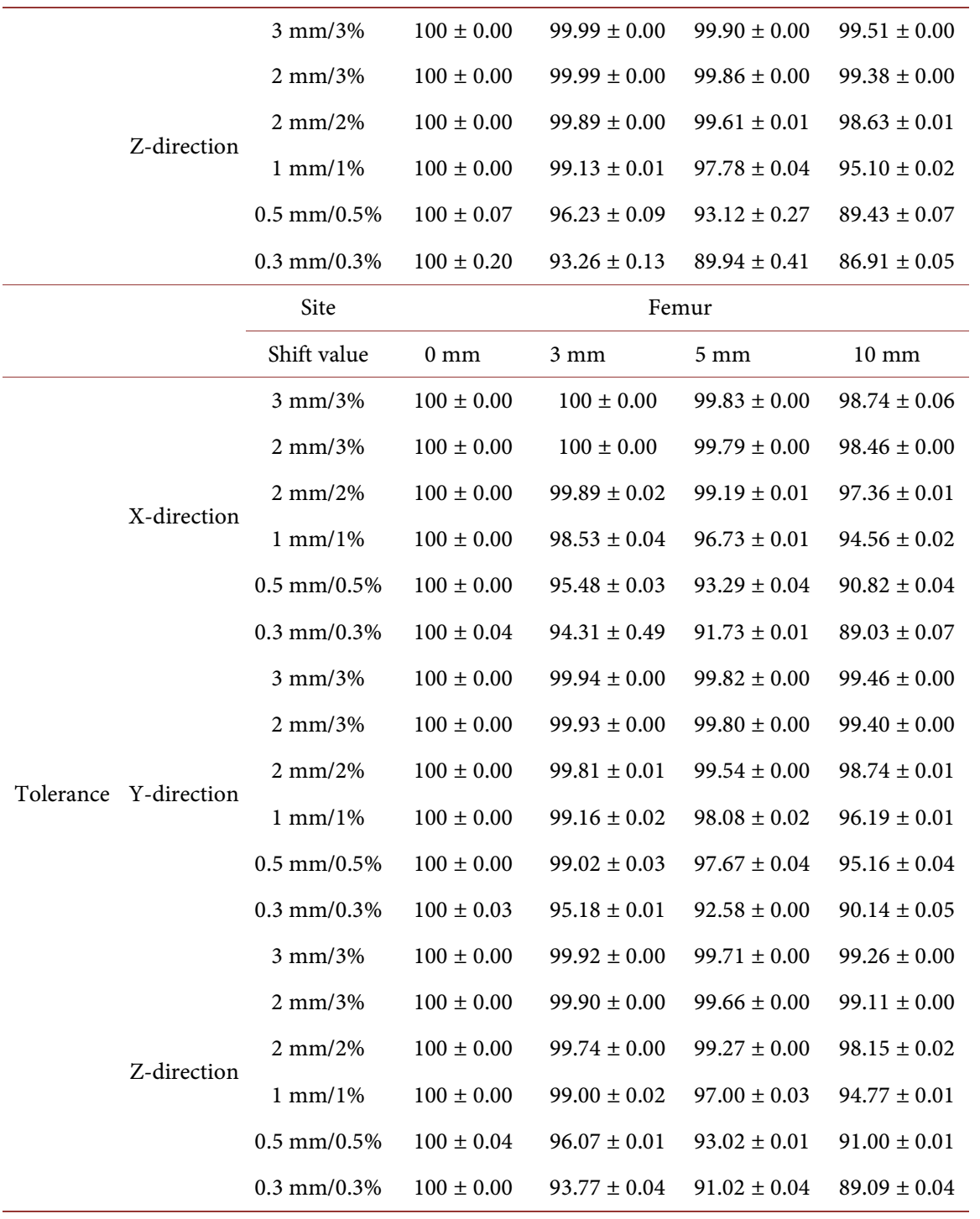

\section{Discussion}

This study showed that body movement can be detected by setting tolerance to $0.3 \mathrm{~mm} / 0.3 \%$ even with $3 \mathrm{~mm}$ shift using in-treatment assessment. In other words, it is possible to detect movement and shape change using in-treatment assessment.

However, in-treatment assessment can automatically send data to a stand-alone workstation when treatment is complete and can then be analyzed. If treatment is not complete, it cannot be analyzed. Therefore, current systems cannot manage in real time. In the future, if a system that can display sinograms in real time and output analysis results simultaneously is implemented, management during treatment in real time will be possible.

If the first reference image is an image that contains many errors, all subsequent data will continue to generate errors. Therefore, in order to use in-treatment assessment, it is considered that selection of a reference image needs attention. 


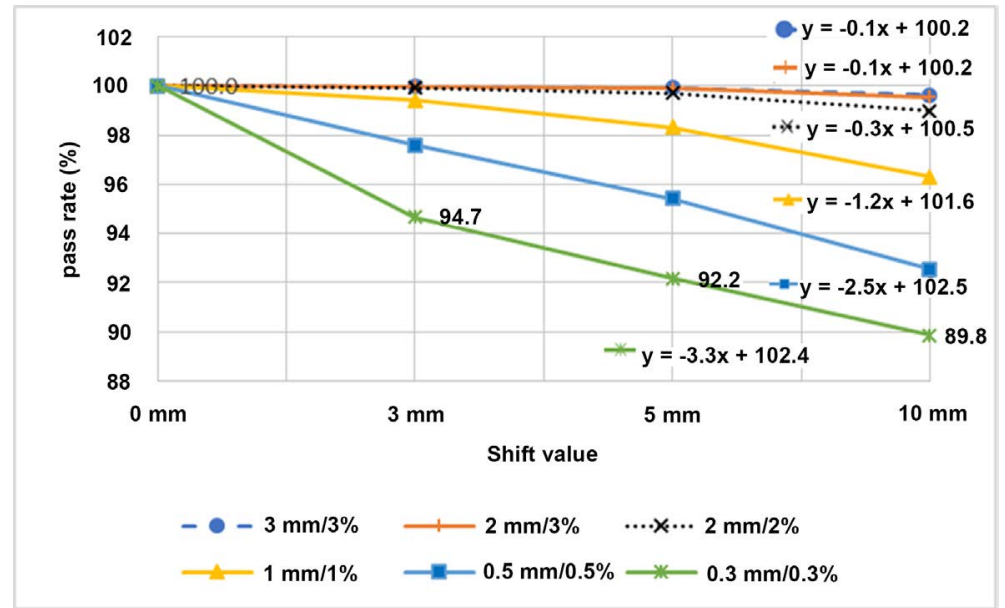

Figure 3. Mean values of gamma analysis at all sites and all directions when the pelvic phantom shift values and tolerance of gamma index were changed.

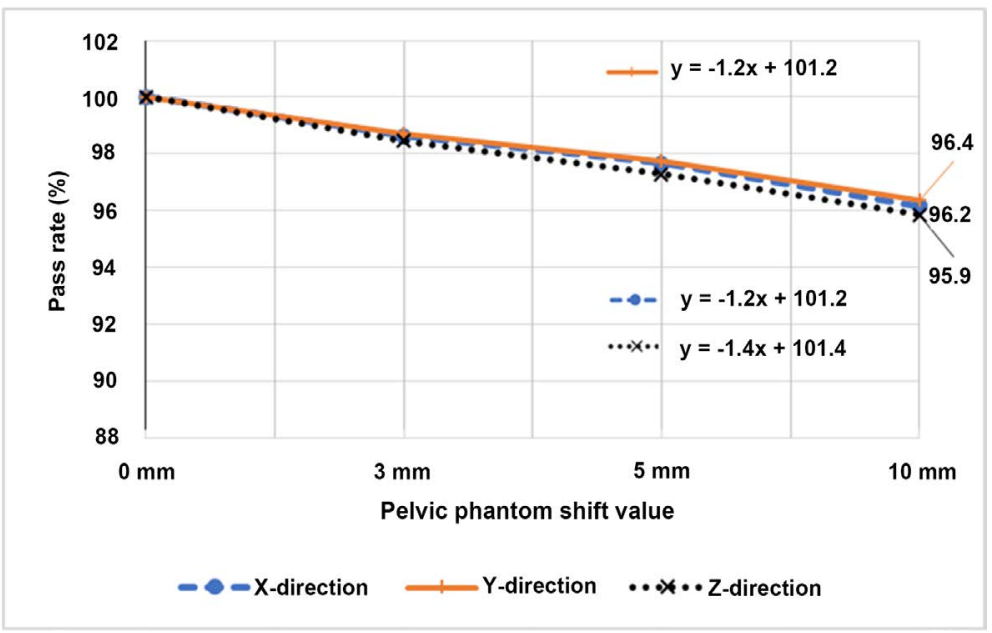

Figure 4. Mean value of gamma analysis at all sites and all tolerance of gamma index when the pelvic phantom shift values and all direction are changed.

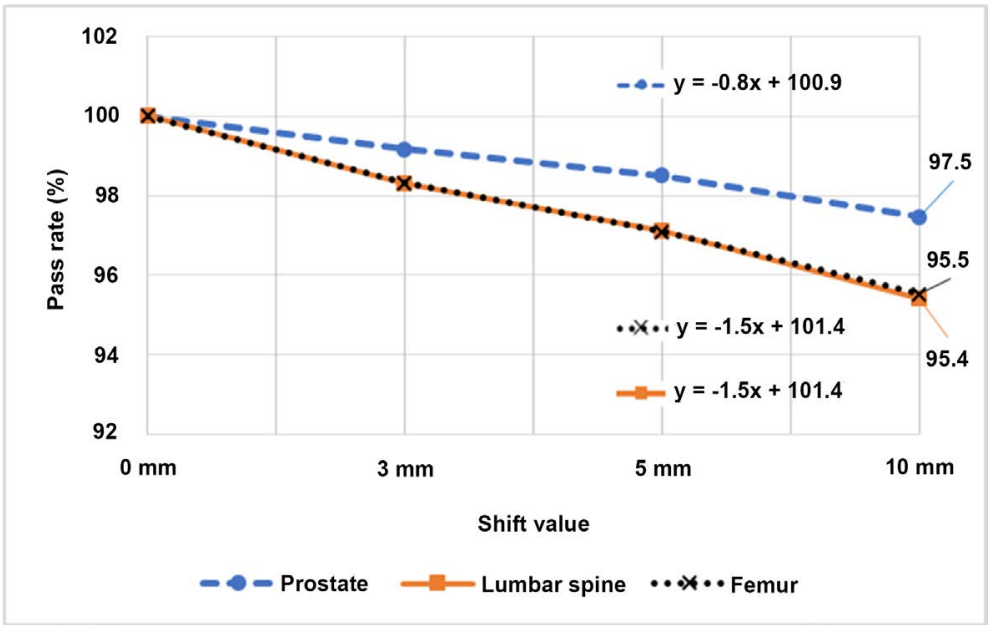

Figure 5. Mean value of gamma analysis in all directions and at all tolerances of gamma index when the pelvic phantom shift values and sites are changed. 
In this study, we used a rigid pelvic phantom. In actual treatment, the patient's body shape may change, and gas in the abdominal cavity may move. This study did not take into account changes in the body shape or gas in the abdominal cavity. Therefore, the analysis results in the clinic will include more uncertainty, and it is expected that a larger error will occur. Therefore, in the future, it is necessary to evaluate during treatment of body shape changes from clinical data, not phantom studies.

This in-treatment assessment confirms the variation from the first reference sinogram. Additionally, a system is also being developed to calculate and evaluate the dose distribution in the body using in vivo calculated images that have been generated [24]. In this software, the sinogram is deconvoluted by a kernel specifically adapted to the MVCT detector to obtain the corresponding primary treatment fluence map. Using this, a dose distribution in the body is created from the planned CT image of the patient. It is also necessary to predict clinical impact while using such software simultaneously.

In this study, we proved that using in-treatment assessment can capture movement during treatment. In the future, if a system that can display sinograms in real time and output analysis results simultaneously is implemented, management during treatment in real time will be possible. If possible, the uncertainty during treatment can be reduced and reliable radiation therapy can be performed.

\section{Conclusion}

In-treatment assessment provides high detection sensitivity, and is a powerful tool that can manage body movement during treatment.

\section{Acknowledgements}

We would like to thank DMC Corp. (https://dmed.co.jp/) for English language editing.

\section{Conflicts of Interest}

The authors declare no conflicts of interest regarding the publication of this paper.

\section{References}

[1] Staffurth, J. (2010) Radiotherapy Development Board. A Review of the Clinical Evidence for Intensity-Modulated Radiotherapy. Clinical Oncology, 22, 643-657. https://doi.org/10.1016/j.clon.2010.06.013

[2] Viani, G.A., Viana, B.S., Martin, J.E., Rossi, B.T., Zuliani, G. and Stefano, E.J. (2016) Intensity-Modulated Radiotherapy Reduces Toxicity with Similar Biochemical Control Compared with 3-Dimensional Conformal Radiotherapy for Prostate Cancer: A Randomized Clinical Trial. Cancer, 122, 2004-2011. https://doi.org/10.1002/cncr.29983

[3] Mackie, T.R., Holmes, T., Swerdloff, S., et al. (1993) Tomotherapy: A New Concept 
for the Delivery of Dynamic Conformal Radiotherapy. Medical Physics, 20, 1709-1719. https://doi.org/10.1118/1.596958

[4] Feygelman, V., Opp, D., Javedan, K., Saini, A.J. and Zhang, G. (2010) Evaluation of a 3D Diode Array Dosimeter for Helical Tomotherapy Delivery QA. Medical Dosimetry, 35, 324-329. https://doi.org/10.1016/j.meddos.2009.10.007

[5] Myers, P., Stathakis, S., Gutierrez, A.N., Esquivel, C., Mavroidis, P. and Papanikolaou, N. (2012) Evaluation of PTW Seven29 for Tomotherapy Patient-Specific Quality Assurance and Comparison with ScandiDos Delta. Journal of Medical Physics, 37, 72-80. https://doi.org/10.4103/0971-6203.94741

[6] Defoor, D.L., Vazquez-Quino, L.A., Mavroidis, P., Papanikolaou, N. and Stathakis, S. (2015) Anatomy-Based, Patient-Specific VMAT QA Using EPID or MLC Log Files. Journal of Applied Clinical Medical Physics, 16, 206-215.

https://doi.org/10.1120/jacmp.v16i3.5283

[7] Mijnheer, B., Olaciregui-Ruiz, I., Rozendaal, R., Spreeuw, H., van Herk, M. and Mans, A. (2015) Current Status of 3D EPID-Based in Vivo Dosimetry in The Netherlands Cancer Institute. Journal of Physics. Conference Series, 573, Article ID: 012014. https://doi.org/10.1088/1742-6596/573/1/012014

[8] Rowshanfarzad, P., Mcgarry, C.K., Barnes, M.P., Sabet, M. and Ebert, M.A. (2014) An EPID-Based Method for Comprehensive Verification of Gantry EPID and the MLC Carriage Positional Accuracy in Varian Linacs during Arc Treatments. Radiation Oncology, 9, 249. https://doi.org/10.1186/s13014-014-0249-8

[9] Bojechko, C., Phillps, M., Kalet, A. and Ford, E.C. (2015) A Quantification of the Effectiveness of EPID Dosimetry and Software-Based Plan Verification Systems in Detecting Incidents in Radiotherapy. Medical Physics, 42, 5363-5369. https://doi.org/10.1118/1.4928601

[10] Vazquez Quino, L.A., Chen, X., Fitzpatrick, M., Shi, C., Stathakis, S., Gutierrez, A., et al. (2014) Patient Specific Pre-Treatment QA Verification Using an EPID Approach. Technology in Cancer Research \& Treatment, 13, 1-10. https://doi.org/10.7785/tcrt.2012.500351

[11] Huang, G., Medlam, G., Lee, J., Billingsley, S., Bissonnette, J.P., Ringash, J., et al. (2005) Error in the Delivery of Radiation Therapy: Results of a Quality Assurance Review. International Journal of Radiation Oncology, Biology, Physics, 61, 1590-1595. https://doi.org/10.1016/j.ijrobp.2004.10.017

[12] Mans, A., Wendling, M., McDermott, L.N., Sonke, J.J., Tielenburg, R., Vijlbrief, R., et al. (2010) Catching Errors with in Vivo EPID Dosimetry. Medical Physics, 37, 2638-2644. https://doi.org/10.1118/1.3397807

[13] Patton, G.A., Gaffney, D.K. and Moeller, J.H. (2003) Facilitation of Radio Therapeutic Error by Computerized Record and Verify Systems. International Journal of Radiation Oncology, Biology, Physics, 56, 50-57. https://doi.org/10.1016/S0360-3016(02)04418-8

[14] Wendling, M., McDermott, L.N., Mans, A., Sonke, J.J., van Herk, M. and Mijnheer, B.J. (2009) A Simple Back Projection Algorithm for 3D in Vivo EPID Dosimetry of IMRT Treatments. Medical Physics, 36, 3310-3321. https://doi.org/10.1118/1.3148482

[15] Mans, A., Remeijer, P., Olaciregui-Ruiz, I., Wendling, M., Sonke, J.J., Mijnheer, B., et al. (2010) 3D Dosimetric Verification of Volumetric-Modulated Arc Therapy by Portal Dosimetry. Radiotherapy \& Oncology, 94, 181-187. https://doi.org/10.1016/j.radonc.2009.12.020

[16] Olaciregui-Ruiz, I., Rozendaal, R., Mijnheer, B., van Herk, M. and Mans, A. (2013) 
Automatic in Vivo Portal Dosimetry of All Treatments. Physics in Medicine \& Biology, 58, 8253-8264. https://doi.org/10.1088/0031-9155/58/22/8253

[17] Fidanzio, A., Azario, L., Greco, F., Cilla, S. and Piermattei, A. (2015) Routine EPID in Vivo Dosimetry in a Reference Point for Conformal Radiotherapy Treatments. Physics in Medicine \& Biology, 60, N141-N150. https://doi.org/10.1088/0031-9155/60/8/N141

[18] Rozendaal, R.A., Mijnheer, B.J., Hamming-Vrieze, O., Mans, A. and van Herk, M. (2015) Impact of Daily Anatomical Changes on EPID-Based in Vivo Dosimetry of VMAT Treatments of Head-and-Neck Cancer. Radiotherapy \& Oncology, 116, 70-74. https://doi.org/10.1016/j.radonc.2015.05.020

[19] Bailey, D.W., Kumaraswamy, L., Bakhtiari, M., Malhotra, H.K. and Podgorsak, M.B. (2012) EPID Dosimetry for Pretreatment Quality Assurance with Two Commercial Systems. Journal of Applied Clinical Medical Physics, 13, 82-99. https://doi.org/10.1120/jacmp.v13i4.3736

[20] Royer, P., Marchesi, V., Rousseau, V., Buchheit, I., Wolf, D., Peiffert, D., et al. (2014) Evaluation of Transit in Vivo Dosimetry Using Portal Imaging and Comparison with Measurements Using Diodes. Cancer/ Radiothérapie, 18, 183-190. https://doi.org/10.1016/j.canrad.2014.03.006

[21] Ford, E.C., Terezakis, S., Souranus, A., Harris, K., Gay, H. and Mutic, S. (2012) Quality Control Quantification (QCQ): A Tool to Measure the Value of Quality Control Checks in Radiation Oncology. International Journal of Radiation Oncology, Biology, Physics, 84, e263-e269. https://doi.org/10.1016/j.ijrobp.2012.04.036

[22] Tarutani, K., Tanooka, M., et al. (2019) Improved Error Detection Using a Divided Treatment Plan in Volume Modulated Arc Therapy. Reports of Practical Oncology and Radiotherapy, 24, 133-141.

[23] Low, D.A. and Dempsey, J.F. (2003) Evaluation of the Gamma Dose Distribution Comparison Method. Medical Physics, 30, 2455-2464. https://doi.org/10.1118/1.1598711

[24] Chung, E., Kwon, D., Park, T., Kang, H. and Chung, Y. (2018) Clinical Implementation of Dosimetry Check ${ }^{\mathrm{Tm}}$ for TomoTherapy ${ }^{\otimes}$ Delivery Quality Assurance. Journal of Applied Clinical Medical Physics, 19, 193-199.

https://doi.org/10.1002/acm2.12480 